\title{
The Norwegian Mother and Child Cohort Study (MoBa) - its birth and early development
}

\author{
Rannveig Nordhagen and Kari Kveim Lie \\ Norwegian Institute of Public Health, Oslo, Norway \\ E-mail: rannveig.nordhagen@fhi.no
}

This is an open access article distributed under the Creative Commons Attribution Licence, which permits unrestricted use, distribution, and reproduction in any medium, provided the original work is properly cited.

\section{THE SHORT VERSION}

The idea of a Norwegian pregnancy cohort was conceived in the early 1990s - in two research centers: The Medical Birth Registry of Norway (MBRN) in Bergen, by Lorentz M. Irgens - initially as an extension of the Medical Birth Registry - and at the National Institute of Public Health (NIPH) in Oslo, by Leiv S. Bakketeig, who suggested a cohort study. The first discussions between researchers from the two centers were informal.

A similar project was planned in Denmark. A fruitful collaboration between the Danish and Norwegian groups was initiated, aiming at future use of data from both projects together. The two projects were also given the same name: "Better Health for Mother and Child," a name the Danish project still carries. However, in spite of good communication between the groups, certain differences in data collection became necessary.

In 1994, the Norwegian project was formalized with Bakketeig as principal investigator, in close collaboration with Irgens. A steering committee was established with the director of NIPH, Bodolf Hareide, as chairman and Rannveig Nordhagen as secretary. There was also a working group with members from Bergen and Oslo, and a reference group, with members representing various fields of research and groups of health professionals.

The first research protocol implicated recruitment of pregnant women at the first visit to their family doctor for antenatal care, as in Denmark. Applications were submitted to the Regional Committee for Medical Research Ethics and to the Data Inspectorate. Both approved the project. The project was also discussed in a government conference in June 1997.

A pilot project was conducted in 1997 in the municipalities Sund and Fjell outside Bergen. The pregnant women were recruited by their general practitioners. The doctors were invited to information meetings with members from the research group. Collaboration was easy, and the atmosphere during the meetings was amiable.

However, some influential family doctors, who previously had expressed concern about ethical aspects of the project, now turned to the Parliament's social committee with their considerations. The committee asked the government to stop the project until these issues were properly discussed. Hence, further work in the project was halted.
During the spring of 1998, detailed information about the project and the questions raised was given to the Ministry of Health and Social Affairs, and to the social committee. After discussions in Parliament, the project was approved. Project work started again!

Bakketeig, tired of the slow progress in the project, was offered a professorship at the University in Odense, and moved to Denmark. Per Magnus, head of the Department of Epidemiology at NIPH, took over as principal investigator.

A new protocol was developed, and various changes were made: The project got a new name, new logistics, and new questionnaires were constructed. This was presented to Regional Committee for Medical Research Ethics and to the Data Inspectorate, and was approved. The new name was: the Norwegian Mother and Child Cohort Study (MoBa). Pregnant women were now to be recruited through the hospital ultrasound clinics, where almost all women in Norway come for routine examination.

Postal questionnaires were for economic reasons chosen instead of telephone interviews, as was used in Denmark, and initially had been planned for MoBa. Questionnaires used in the pilot study were revised. A new dietary questionnaire was constructed, and questionnaires for follow-up after birth were planned.

MBRN was responsible for sending out and receiving questionnaires, for checking of the data, and for construction of research files. Moreover, a tracking system was constructed - with names and addresses of participants, kept separated from the information collected from them. Privacy was strictly ensured in all work.

NIPH took responsibility for the construction of a biobank for long term storage of biological samples, and a system for retrieval of the samples. Moreover, standardized procedures for blood sampling, shipping and processing of specimens were established, and distributed to the hospitals together with standardized equipment.

In the yearly allocation of resources and tasks to NIPH, 1 million NOK was supplied to the project for 1998, and again for 1999. However, it was stated that NIPH should be responsible for the general funding of the project. The cost for nationwide implementation was estimated to 20 million NOK annually. Since this funding was not within reach, recruitment had to be stepwise. In June 1999, recruitment of pregnant women through ultrasound clinics started at Haukeland University Hospital in Bergen. In the months and years to 
come, recruitment started also in other hospitals.

From 2000 and onwards, fathers-to-be were included in the study - by invitation through their pregnant partners. Blood samples from fathers were obtained at the ultrasound laboratories, since most fathers-to-be accompany their partner to this examination. About $80 \%$ of the fathers of consenting partners agreed to participate.

In 2002, the recruitment became nationwide, and from 2005 and onwards all hospitals with more than 200 births annually participated except Rikshospitalet in Oslo and the University Hospital in Tromsø, where other projects that recruited pregnant mothers were ongoing. The recruitment period lasted for almost 10 years, until December 2008.

\section{THE EARLY STAGE}

During the 1990s, the NIPH in Oslo was going through major changes. Several of the routine laboratory activities, which until then had been centralized, were set out to regional and local laboratories, and more focus was placed on research. New fields, such as epidemiology, community medicine and child health came on the agenda. It was under these circumstances that the idea of a large cohort study, following the child from prenatal life through childhood and adolescence into adulthood, came forward. Also, at the MBRN, which in those days was organized under the University of Bergen, scientists were working with perinatal epidemiology. MBRN was established in 1967 in order to monitor adverse birth outcomes and is by law receiving information from attending midwifes and physicians for every birth in Norway. The report includes data from the pregnancy. Although already a gold mine for epidemiological research, supplemental information about pregnancy exposures and child health would immensely enrich the research potential in MBRN. This was discussed among the Bergen scientists in the 1990 s, who realized that consent based data collection was necessary, and the idea of a cohort study came up $(1,2)$. Thus, the pioneers of a nationwide cohort study starting in fetal life were Bakketeig, Irgens, Hareide and Johannes Wiik, the director of the Department for Prevention and Health Promotion at NIPH. Preliminary discussions were informal, and were between these people and several others interested in the project. The administrative foundation of the project was from 1993 in Wiik's department, but later, from 1996, the project was organized directly under the director of NIPH. From 2002, MBRN was organized as a department at NIPH, which facilitated the collaboration.

The idea was to collect information from pregnant women about a range of exposures, and afterwards follow the mothers and their children through birth and onwards. In this way, multiple studies on the relationship between exposures and a large number of health outcomes could be performed. In the early 1990s, this was innovative, and many people - also scientists argued that it was too large and expensive, and pointed to more tailored studies. Anyhow, a short, preliminary protocol was presented to The Ministry of Health and to the Directorate of Health. Many research questions were raised, for instance: Could environmental toxicants harm the fetus? Do subclinical infections during pregnancy (recognizable in the blood specimens) give rise to congenital malformations or developmental disorders? Is use of mobile phones harmful? Could the data collected throw light on the fetal origins of adult health (the Forsdahl-Barkerhypothesis)? Although the project was well received, no additional funding was available and further work on the project was dependent on allocation of workforce already at MBRN and NIPH.

\section{Collaboration with DenMaRK}

In Denmark a similar project was planned. A broad coalition of Nordic cohorts was also discussed, but was not realized. However, a long-term collaboration between the Danish and the Norwegian project groups was established, and several meetings were held - the first in January 1992. The intention was to facilitate future use of data from the two studies combined in research projects. In June 1994, the collaboration became more formalized, and people dedicated to various aspects of the studies were appointed. The two sister projects were given the same name: "Better Health for Mother and Child". For economic reasons some differences in logistics, especially concerning data collection, emerged $(3,4)$.

\section{ORgaNIZATION}

At the meeting in 1994, mentioned above, further work with the project in Norway became more formalized. Bakketeig was appointed principal investigator, working in close collaboration with Irgens. A steering group was established with the following members: Hareide (chairman), Bakketeig, Irgens, Wiik, Erik Dybing, Ivar Ørstavik and Rannveig Nordhagen (secretary). A working group of people from Bergen and Oslo was established: From MBRN: Pat Schreuder, Rolv Terje Lie, Stein Emil Vollset, Rolv Skjærven and Kjell Haug. From NIPH: Rannveig Nordhagen, Kari Kveim Lie, Anne Eskild and Per Magnus. Later, many others joined the work force: Kjersti Skjold Rønningen, who established the biobank, Helle Meltzer, who designed a new food frequency questionnaire, Ragnhild Hovengen and Jorid Eide, who primarily were working with recruitment and collaboration with hospitals, and Arild Sunde and Elin Alsaker, who built up systems for tracking of participants and for data cleaning and storage. An external reference group was established, with the purpose of obtaining input on design and data collection, as well as to give information about the project, and hopefully to evoke understanding and enthusiasm among various research groups and health professionals. At the first meeting, on January 25, 1995 Hareide presented the project, and emphasized the need for advice in order to develop a good project. However, in 2002, when the recruitment procedures 
were well established, the formal reference group was discontinued, and ad hoc groups were established for input to the construction of questionnaires.

\section{PROTOCOL AND APPROVALS}

The first research protocol was drafted with a design very similar to the Danish project. The plan was to recruit pregnant women at their first visit for antenatal care. After the general practitioner had given information, women who wanted to participate were asked to sign the consent form, and a blood sample for the project was collected along with routine sampling. The blood samples were to be sent to the NIPH. The women also would be asked to give information about health and lifestyles - in questionnaires or through a telephone interview. Birth information would be obtained from the MBRN, while a blood sample from the mother and cord blood from the child would be collected at the maternity ward and sent to NIPH. Applications and the protocol were sent to the Regional Committee for Medical Research Ethics and to the Data Inspectorate. The project was approved by both these agencies, and a pilot study was planned.

\section{Pilot STUdY}

In 1997 , a pilot study was conducted in the municipalities of Fjell and Sund outside Bergen. General practitioners were invited to meetings with members from the research group. The collaboration with the doctors was generally very good, although some of them were more enthusiastic than others. This was reflected in varying participation rates for pregnant women among doctors. The overall attendance rate for women in the pilot project was high, about $80 \%$ of invited women. However, we realized that it would be difficult to keep in such close contact with the staff involved if recruitment should take place in all municipalities in the country.

\section{DISCUSSIONS WITH PROFESSIONALS}

To generate interest for the project and possibly prevent negative attitudes among professionals who could come in contact with pregnant women, various groups and representatives from professional organizations were invited to information meetings and discussions. The midwives had, and still have, two organizations: The Norwegian Association of Midwives, and The Midwifery Association - the latter of which is a subdivision of the Norwegian Nursing Association. Representatives from both organizations were invited. The collaboration with midwives - at this stage, as well as during the years of recruitment, was positive and met with few difficulties - it seemed that the vast majority of this group recognized the importance of the project. Also, representatives of the hospital laboratory staff were invited to information meetings. They would be responsible for blood sampling prenatally as well as after birth. Collaboration with this group was also pleasant. Although collaboration with family doctors in the pilot project was very good, some other general practitioners were more skeptical: Was it worthwhile? Was potential anxiety among the participants sufficiently taken care of? Moreover, they pointed out that recruitment of pregnant women meant extra work for the doctors, without any compensation offered.

\section{ETHICAL QUESTIONS}

In 1995, a conference on ethical aspects of the project was held at NIPH. Epidemiologists and representatives from various medical fields, including medical ethics, were invited from various parts of the country. Above all it was emphasized that benefits always had to be greater than the risk imposed on the participants. As the project was an observational study without intervention, the risk was considered very small. Blood samples would admittedly be taken, but together with the routine samples in antenatal care by use of socalled multiple sampling, i.e. only one venous puncture and several samples for assorted purposes. Hence, this risk was considered non-significant. Although discussions were lively, at the end of the day the conclusion was that the project is important and should be realized. The following topics for ethical discussions were brought up at the conference, and were also discussed in the project groups, and at information meetings as well as with formal bodies:

\section{Can the mother give consent on behalf of her unborn} child?

This was initially considered acceptable, but later it was established that participating children must be informed about their participation at age 15, and asked to give their own consent when they are 18 years.

\section{Is informed consent meaningful when plans for future use of collected data are so vague?}

Typically, an informed consent requires that research questions are described precisely. What does informed mean in our context? How can the participant possibly be informed about issues that later might be studied in the light of new knowledge, or by use of technology developed in the future? This challenge was met by great emphasis on information about various aspects of the study, yearly newsletter to participants about ongoing research, and by keeping an updated project website. Above all, a participant must be able to withdraw at any time, without giving any reason.

\section{Should participants be informed about laboratory findings?}

What if laboratory analyses reveal signs of a disorder where intervention is indicated? What if the participant specifically asks for it? In the consent form it is clearly stated that results will not routinely be reported back to the participants.

\section{How should potential nonpaternity be handled?}

Paternity testing will be a consequence of genetic analyses. This information must never be disclosed. 
Would all the focus on risk factors and diseases in the questionnaires lead to anxiety among pregnant women?

This concern was raised at an early stage, especially by some family doctors. During several information meetings with professionals who were working with pregnant mothers, we had discussions preparing for this possibility, and also welcomed telephone calls for further discussions. Moreover, in all letters to the participants, including the questionnaires, participants were welcomed to call the working groups in Bergen and Oslo, who would answer questions.

\section{External ethics group established in 1997}

An external ethics group was established in February 1997. Group members were: Per Bergsjø (Chairman), Reidun Førde, Roar Johnsen and Jan Helge Solbakk. However, the group found that communication with the project was insufficient, and perceived that they were not consulted at an early enough stage, especially since changes in the protocol were made without involving the group. Hence, the group resigned. Much of this was probably because changes were made as a result of negotiations with the Regional Ethics Committee, and were not discussed in parallel with the ethics group.

\section{THE PROJECT WAS HALTED}

During the fall of 1997, objections from general practitioners escalated and were brought to the newspapers. Some influential general practitioners also contacted representatives of the Parliament's Committee for Social Affairs, and expressed their doubts about the project's usefulness and their concerns over ethical issues. The Social Committee asked the government to stop the project until these issues were properly clarified and discussed.

\section{A NeW START}

In the spring of 1998, the Ministry of Health and Social Affairs informed the Parliament about the project and the concerns that had been raised. After the debate, an overwhelming majority voted in favor of the study. Thus, project work continued. A new protocol was written. The main change was recruitment through ultrasound laboratories. The change was approved by the Regional Ethics Committee and the Data Inspectorate. In the meantime, Bakketeig, tired of the slow progress in the project, had been offered a professorship at the University in Odense and moved to Denmark. Per Magnus, head of the Department of Epidemiology at NIPH, took over as the new principal investigator. People at MBRN was, as previously, responsible for sending out and receiving the questionnaires, for scanning and quality check of the questionnaires, and for storing data and establishing research files, under the leadership of Pat Schreuder. Furthermore, a tracking system was developed, to update all events, and to send out questionnaires and information letters at the right points in time (5). People at NIPH were responsible for developing the biobank and for sending out standardized vials and sampling procedures $(6,7)$. Regarding collaboration with the recruiting hospitals, MBRN the was primary contact for hospitals in the western counties, while NIPH was primary contact for the other counties.

\section{NeW NAME}

It had been argued that the project name Better Health for Mother and Child could give false hopes for better health simply by consenting to participate, while the reality was a hope for better health in the future, as a result of research. The name was therefore changed to the more descriptive The Norwegian Mother and Child Cohort Study (MoBa).

\section{RECRUITMENT BY ULTRASOUND LABORATORIES}

It would clearly have been demanding to follow up all the general practitioners in the country in the same way as we did in the pilot study. Moreover, major criticism had been raised among groups of family doctors, which could create considerable difficulties. Hence, we explored other logistics. Kjell Haug suggested that pregnant women should be recruited through the ultrasound laboratories, a strategy which was approved by the working group and also by the steering group (8). As routine ultrasound takes place around pregnancy week 18, this change in logistics meant that most women had come a bit further in their pregnancy at recruitment compared to the logistics used in the pilot study. Therefore, questionnaire information about the first pregnancy weeks might suffer from more recall bias. It also meant that the first blood sample was drawn in second trimester instead of during the first trimester. However, this seemed to be a necessary compromise. Since more than $95 \%$ of pregnant women attend this routine ultrasound at governmental hospitals, there was little reason to believe that the change would lead to social selection. We were happy that a few private specialists, who performed ultrasound examination in their clinics, volunteered to recruit pregnant women to MoBa, following basically the same the same procedures as recruitment in the hospitals.

\section{RECRUITMENT PROCEDURES}

1. When a pregnant woman received a letter from the ultrasound clinic with appointment for routine antenatal ultrasound examination, she was also briefly informed about MoBa. This was meant to show the women that the hospital approved the study.

2. Lists of names and addresses of women who were scheduled for routine ultrasound examination in the coming weeks were regularly sent to MBRN from the hospitals' IT departments or central offices.

3. Based on these lists, the project sent an information brochure and consent form to women prior to the ultrasound examination (later also to their partners). 
4. At the ultrasound laboratory the women would meet a midwife, who could give information about the project and answer questions from the women.

5. Blood samples and urine samples were collected from consenting women (later also from fathers) at the same visit. Samples were processed and packaged at the hospital laboratories, and shipped to the biobank at NIPH.

6. A copy of the findings at the ultrasound examination was later sent to MBRN.

7. After birth, another blood sample from the mother and a cord blood sample from the child were taken, and were shipped to the biobank at NIPH.

\section{ESTABLISHING COLLABORATION WITH THE HOSPITALS}

In Norway, there were 52 hospitals (all governmental) with more than 200 births per year. We contacted them one by one, and 50 of them became our partners in recruiting participants to MoBa. In addition, we appreciated that some smaller clinics and a few private specialists, who performed ultrasound examinations in their clinics, volunteered to participate in the recruitment. As the first step the hospital owner, in those days the county administrations, was contacted and informed about the project - often through personal meetings. Next, the hospital's chief administrative leader was contacted. Hareide took part in this work. He was a valuable door opener for the project due to his contacts with county administrations and people in the health system. When everything was approved from these authorities, contact was made with chief medical officers/heads of department at relevant departments in the hospitals (ultrasound clinics, obstetric wards, laboratories, and IT departments or office services). All links in this chain had to consent in order to manage the procedures listed above. Members of the working group travelled to meet with all these persons, in order to give information and to negotiate about practical issues. Ragnhild Hovengen, as a profiled health nurse and Jorid Eide, as an experienced midwife, established particularly good contact with partners in the hospitals. However, we all were met with great interest and goodwill during meetings in the hospitals.

It was a challenge that the structure and organization varied quite widely from one hospital to the other. In particular, this applied to the lists of names and addresses of the women - a prerequisite for inviting them to MoBa. In several hospitals, the lists were worked out electronically by collaboration between IT staff in the hospitals and the IT group at MBRN. But not all hospitals had procedures to do this, and manual lists had to be written and sent by surface mail.

Another problem was that some hospitals were already recruiting pregnant women to other research projects. Therefore, recruitment to our project was postponed in those hospitals. For this reason, Ullevål university hospital was one of the last hospitals to be included. In some of the larger hospitals the project paid the salary, or part of the salary for a person as compensation for the work to recruit women. But overall there was little to spend on compensation to the hospitals. However, we promised to provide input through information meetings and give lectures on related themes, as well as to give information about progress and findings in the project. This was in the interest of the project, because it was important that midwives and other staff, who had contact with pregnant women, were well informed - and hopefully positive to MoBa. This meant more travels and more lectures for members of the working group. Moreover, information letters were regularly sent from the project, and later annual MoBa newsletters were sent to participating hospitals as well as to the participating women. The information letter "Birth News from MBRN", which is sent to all maternity wards in the country, also brings news from the project.

Recruiting 100,000 women was ambitious, and required a lot of work, lasting several years in most hospitals. During all this time hospital staff made a considerable contribution to the project. Everyone did a wonderful job, but without the skilled and enthusiastic participation from the midwives, MoBa would never have been the success it has turned out to be.

\section{FATHERS INCLUDED IN THE STUDY}

Early in the project, researchers as well as participating mothers, pointed out that also fathers ought to participate in the study. This would provide opportunities to study potential effects of father's occupational exposure, drug use, life styles etc., and also to do genetic analyses. In May 2000, an application was submitted to the Regional Ethics Committee and to the Data Inspectorate, and they both were positive. Fathers were included and invited via their pregnant partners. Blood samples from fathers were obtained at the visit to the ultrasound clinics, as most fathers-to-be accompany their partners to this examination. About $80 \%$ of the fathers participate.

\section{RECRUITMENT LASTED FOR TEN YEARS}

In June 1999, recruitment started at Haukeland University Hospital in Bergen. In the months and years to come the project expanded, and by 2002, we were recruiting women from all parts of the country. The recruitment period lasted for ten years - from June 1999 through December 2008. The reason for using so many years to recruit the women was primarily the limited funding, which made it impossible to approach more than a few hospitals at the same time, but also because the participation rate was lower than we had expected (about 40\%). After recruitment was completed, the project proceeded with follow-up and new data collection from the participants (4).

\section{THE QUESTIONNAIRES}

In the pilot study, we had tested out questionnaires as well as telephone interviews. The latter was used in the Danish project, and we had hoped to get a similar app- 
ointment with the telephone company as they had in Denmark. The telephone company, however, had new leaders, and the offer we got was far out of reach for our budgets. Therefore, we relied on postal questionnaires. The questionnaires from the pilot were revised, and new questionnaires were designed.

Three questionnaires were sent out during pregnancy: a general questionnaire on health, disease and life styles at about week 15, a dietary questionnaire at week 22, and again a general questionnaire at week 30 . More questionnaires were sent out at 6,18 and 36 months after birth, and again when the child was 5, 7 and 8 years.

Most questions were designed after discussions with colleagues and researchers in different disciplines and in various medical specialties. Some researchers also contacted the project and provided input to the questionnaires. A special challenge was that several areas could not be elucidated by a few simple questions, but might require a series of questions, and preferably be based on validated measures, published by others.

As large expenses were required to print, send out and receive questionnaires for a cohort of 100,000 participants, some of the questionnaires were funded by specific grants, and were therefore characterized by special interest questions, such as developmental disorders, asthma and mental health. Not only the topic, but also the wording of the questions and of the answering alternatives had to be discussed. Regardless of funding, the questionnaires were always discussed in the working group, and had to be approved by the steering committee before they were sent out. Within the working group there were differences in background and in the field of professional interest, an advantage in many ways, but certainly a challenge in reaching agreement.

Instead of piloting the questionnaires, an evaluation was performed after a certain numbers of questionnaires had been received, followed by a revision of the questionnaire - a fact that researchers using the data must take into consideration. These revisions were also vigorously discussed. The wording in some of the standardized batteries was not always well received by the participants, and a debate of whether the original wording should be continued or not, took place. Further, continuity across questionnaires versus including new areas was discussed. The most difficult task, however, was to limit the number of questions.

Seminars were held, one of them discussing mental health and child development. Researchers from many institutions were invited. This was the start of a close collaboration - and lengthy discussions - with Synnve Schjølberg, Kristian Tambs and Espen Røysamb.

A working group was established with representatives from MBRN, NIPH and the Institute for Nutrition Research at the University of Oslo. Members were: Christian A. Drevon, Tore Henriksen, Grethe S. Tell, Jan Alexander and Stein Emil Vollset. A note describing research interests and the collaboration between the institutions was written. Contact was also made with Sjurdur Olsen in the Danish sister project. Initially, MoBa used a dietary questionnaire developed at the University of Oslo - a modified version of the questionnaires used in the "Norkost studies" in the general population. Assuming that women did not change their diet when they become pregnant, the questions were about dietary habits during the year before pregnancy. After discussions, however, the conclusion was that the questionnaire should be designed for pregnant women, with special emphasis on diet after the start of pregnancy. At a meeting in the steering group in June 2001, it was decided to develop and implement a new dietary questionnaire, specifically designed for MoBa. Collaboration with the Institute of Nutrition Research at University of Oslo was then ended. Helle M. Meltzer, and later also Margareta Haugen and Anne Lise Brantsæter at NIPH, were responsible for development and validation of a new dietary questionnaire, which was used from March 2002. The first two years it was sent out together with the first questionnaire in week 15, but was later sent in the 22 nd week of pregnancy.

\section{THE BIOBANK}

One of the biggest challenges in the project was the development of a biobank for processing, storing and retrieving biological samples. This work was led by Kjersti Skjold Rønningen with much help from Liv Paltiel and others. Rannveig Nordhagen, who previously had been a laboratory scientist, also took part in this work. An informal biobank seminar was held, with the following participants: Elaine Gunter, Richard Jones, Mads Melbye, Egil Jellum and Anne-Lise Børresen Dale. Several of the topics below were discussed.

\section{Which samples should be collected?}

Blood samples were taken in standard EDTA-tubes, provided to the hospitals together with instructions for sampling. Ideally, maternal blood from all pregnancy trimesters should have been collected, but for practical reasons we decided on two maternal samples, one in mid-pregnancy, taken at the visit to the ultrasound clinic - and one sample after delivery. Samples from participating fathers were also taken at the ultrasound clinic or, the necessary tubes were sent to the father, who would ask his family doctor to draw the blood sample. Cord blood from the children were collected soon after birth, but if this, for some reason, was not done, capillary blood to the project was taken at the same time as blood was taken to screen for metabolic diseases.

\section{Environmental samples}

In 2002, collaboration with the National Institute of Environmental Health Sciences (NIEHS) in the US was established with the purpose to study potentially harmful effects of environmental toxicants. A urine sample from the mother was useful for such studies, 
and was therefore added, as was also separate test tubes to be used only for analyses of environmental toxicants.

\section{RNA samples}

From early 2005, additional cord blood was collected in Tempus tubes, containing RNAse inhibitors. This makes it possible to study RNA, which otherwise quickly is degraded. This was funded through collaboration with scientists at Columbia University.

\section{How should the samples be processed and shipped to the biobank?}

In order to avoid leakage of certain substances out from blood cells into plasma, one of the test tubes were spun after sampling, and plasma pipetted off other tubes were shipped without processing. Shipping in cooling packages was out of reach for economic reasons, but it turned out that most specimens arrived at the biobank already the next day by regular post, and were in good shape. Moreover, any irregularity in the shipment was listed along with the date when blood was drawn and the date of specimen's arrival at the biobank. Hence this could be taken into account in the analyses.

Upon arrival in the biobank, personal identification tags were removed from the test tubes. The samples were transferred to vials with a code number - in order to protect privacy, and also to facilitate retrieval of samples. A data program was developed for this purpose, and later this was built into the MoBa tracking system developed in Bergen.

\section{Extraction of DNA, aliquoting and storage of the samples}

From all participants DNA was extracted from one of the tubes, and the concentration normalized. All samples were aliquoted into several units for storage. Microplates with small wells were chosen, as the amount of material was relatively limited. These plates, however, had the disadvantage that the entire microplate was thawed when one well was to be retrieved; thus reducing the quality of the other specimens. Later on, plates with wells for individual micro-tubes became available, making it possible to take out one sample without thawing the other samples on the plate.

When the biobank was established, storing at minus $20^{\circ} \mathrm{C}$ was the common procedure for blood banks. However, freezers holding minus $80^{\circ} \mathrm{C}$ were available, and these were chosen for storage of whole blood, plasma and urine. The shelf life of isolated DNA is, however, very long, and therefore DNA could safely be stored in freezers at minus $20^{\circ} \mathrm{C}$. Security procedures were established, and the freezers were also connected to an external alarm system, prepared to cope with potential power failure $(6,7)$.

\section{Retrieval of samples}

A computer program was necessary for the retrieval of samples. This was initially developed specifically for the biobank through a consulting IT company (Tieto
Enator). However, the need for a program combining the biobank and the data collection in Bergen became obvious. Hence, a new program was developed by the staff in Bergen, and was implemented in May 2011.

\section{ProjeCt ECONOMY}

Project work during the first years was funded solely through the regular budgets in NIPH and MBRN, as people involved were spending parts of their working hours on the project. Necessary travelling was also paid for by these institutions. This was possible because of wholehearted support from Hareide, and from later directors (Geir Stene-Larsen and Camilla Stoltenberg). In the meantime, various external sources, such as The Norwegian Research Council (NRC), health authorities, public and private organizations as well as private businesses were approached. The NRC answered that the cost of the infrastructure of such a gigantic project was not within their reach, but invited us to come back with application for specific subprojects. Also, other efforts ended rather fruitless, except the Confederation of Norwegian Enterprises (NHO), that offered some support for 2-3 years. In 1996, an application was sent to National Institute of Child Health and Development (NICHD) in USA, focusing on environmental exposures and congenital malformations. After very encouraging feedback, the application was turned down, when logistics was changed to recruitment later in pregnancy, and no blood samples were provided during the first trimester. Somewhat later, however, good relations with the NIEHS led to a contract that supported the collection and storage of blood and urine samples, and which provided basic support for the data collection procedures.

In 2003, a large research grant for autism studies was awarded from National Institute of Neurological Disorders and Stroke (NINDS) in USA through collaboration with Ian Lipkin and Ezra Susser at Columbia University in New York. The CoPI from Norway was Camilla Stoltenberg. This project also supported extra data collection and clinical examinations of children who screened positive for autism spectrum disorders at the age of 3 years, and controls.

\section{MOBA - ARE THE EXPECTATIONS FULFILLED?}

Of the women invited to participate, only $40 \%$ gave their consent. This means that some limitations must be kept in mind, which are described in two articles, where the main conclusion is that while prevalence studies must be interpreted with great caution, the low participation rate is less problematic for association studies $(9,10)$. Why was the participation rate so low? Was it because the critics were right when they said we scared the pregnant women with so much focus on risk factors and disease? Or did we ask for too much when sending out these long questionnaires? For ethical reasons we were not allowed to approach nonparticipating women and ask these questions. However, in the pilot study we conducted a telephone survey among 
participating women about this topic. From this study, we have no evidence that women became scared because of all the questions. However, several participants have pointed out that it is a pretty big job to fill in the questionnaires. Whatever the reason was for the low participation rate, also other studies, both in Norway and abroad, have experienced lower participation rates during the later years, than 30-40 years ago.

On the other hand, women who had signed the consent form, followed up very well during pregnancy, with response rates above $90 \%$ for the first questionnaires. However, the response rate decreased in the questionnaires after birth, and came down to $60 \%$ in the questionnaire 36 months postpartum. Very few participants have withdrawn from the study and refused further use of their questionnaire data and biological samples.

Upon application and an access fee (see guidelines at www.fhi.no/moba-en), the data and biological samples are available for researchers both within and outside NIPH, as well as for international groups. Access to biobank material is, however, more restricted because of limited material available. As of December 2014, more than 300 scientific papers in international journals are listed in Pubmed, and 30 doctoral dissertations have been defended, based on MoBa data (www.fhi.no/moba-en).

\section{The VALUe OF MoBA WILl CONTINUE TO RISE}

The possibility of linking MoBa to the Norwegian Patient Registry, the Norwegian Prescription Database and other health registries allows for research far beyond what MoBa alone can provide. The combination of biological samples from the mother, father and children, questionnaire data, registry data, and potentials for new data collection certainly is unique. Collaboration with the Danish sister cohort, which will be further developed, will double the number of individuals under study. Other approaches will be used when new knowledge provides new research questions, and the biobank will increasingly be a gold mine as new technology is developed, and when new hypotheses are developed.

\section{THANKS TO THE PARTICIPANTS}

MoBa participants, who have given biological samples to the project and have done a considerable piece of work in answering the lengthy questionnaires, have donated a wonderful gift to society.

\section{REFERENCES}

1. Irgens LM. The background of the Norwegian Mother and Child Cohort Study. Norsk Epidemiologi $2014 ; 24$ (1-2): 7-8.

2. Bakketeig LS. Why did we start the MoBa study? Norsk Epidemiologi 2014; 24 (1-2): 9.

3. Olsen J, Meder IK. Better health for mother and child - The Danish National Birth Cohort (DNBC), its structure, history and aims. Norsk Epidemiologi 2014; 24 (1-2): 37-8.

4. Magnus P, Irgens LM, Haug K, Nystad W, Skjærven R, Stoltenberg C and the MoBa study group. Cohort profile: The Norwegian Mother and Child Cohort Study (MoBa). Int J Epidemiol 2006; 35: 1146-50.

5. Schreuder P, Alsaker E. The Norwegian Mother and Child Cohort Study (MoBa) - MoBa recruitment and logistics. Norsk Epidemiologi 2014; 24 (1-2): 23-7.

6. Rønningen KS, Paltiel L, Meltzer HM, Nordhagen R, Lie KK, Hovengen R, et al. The biobank of the Norwegian Mother and Child Cohort Study: A resource for the next 100 years. Eur J Epidemiol 2006; 21: 619-25.

7. Paltiel L, Haugan A, Skjerden T, Harbak K, Bækken S, Stensrud NK, Knudsen GP, Magnus P. The biobank of the Norwegian Mother and Child Cohort Study - present status. Norsk Epidemiologi 2014; 24 (1-2): 29-35.

8. Haug K, Schreuder P. Choosing a model for recruiting 100,000 pregnant women to the Norwegian Mother and Child Cohort Study (MoBa). Norsk Epidemiologi 2014; 24 (1-2): 13-4.

9. Nilsen RM, Vollset SE, Gjessing HK, Skjærven R, Melve KK, Shreuder P, et al. Self selection and bias in a large prospective pregnancy cohort in Norway. Paediatr Perinatal Epidemiol 2009; 23: 596-608.

10. Nilsen RM, Surén P, Gunnes N, Alsaker ER, Bresnahan M, Hirtz D. Analysis of self-selection bias in a population-based cohort study of autism spectrum disorders. Paediatr Perinat Epidemiol 2013; 27: 553-63. 